\title{
Peregrine Solitons Beyond the Threefold Limit and Their Two-Soliton Interactions
}

\author{
Shihua Chen, ${ }^{1, *}$ Yanlin Ye, ${ }^{1}$ Jose M. Soto-Crespo, ${ }^{2}$ Philippe Grelu, ${ }^{3}$ and Fabio Baronio ${ }^{4, \dagger}$ \\ ${ }^{1}$ School of Physics, Southeast University, Nanjing 211189, China \\ ${ }^{2}$ Instituto de Óptica, Consejo Superior de Investigaciones Científicas (CSIC), Serrano 121, Madrid 28006, Spain \\ ${ }^{3}$ Laboratoire ICB, U.M.R. 6303 C.N.R.S., Université Bourgogne Franche-Comté, 9 avenue A. Savary, F-21078 Dijon, France \\ ${ }^{4}$ INO CNR and DII, Università di Brescia, Via Branze 38, 25123 Brescia, Italy
}

(Received 6 May 2018; published 6 September 2018)

\begin{abstract}
Within the coupled Fokas-Lenells equations framework, we show explicitly that, in contrast to the expected threefold-amplitude magnification, Peregrine solitons can reach a peak amplitude as high as 5 times the background level. Besides, the interaction of two such anomalous Peregrine solitons can generate a spikelike rogue wave of extremely high peak amplitude, depending on the parameters used. We numerically confirm that the Peregrine soliton beyond the threefold limit can be reproduced from either a deterministic initial profile or a chaotic background field, hence anticipating the feasibility of its experimental observation.
\end{abstract}

DOI: 10.1103/PhysRevLett.121.104101

The Peregrine soliton (PS) concept, as a paradigm of coherent structures in modeling extreme wave events [1,2], is attracting great interest in a variety of scientific areas ranging from hydrodynamics and oceanography to nonlinear optics [3-5]. It was first discovered in 1983 by Peregrine when looking for surface waves in deep water modeled by the nonlinear Schrödinger (NLS) equation [6], hence its current name. Afterwards, its dynamics were seen in a number of physical settings, first in nonlinear fibers [7], then in water wave tanks [8] and plasmas [9], and recently in an irregular oceanic sea state [10]. Contrarily to the usual shape-invariant soliton [11], the PS, basically expressed by a rational function of second degree, presents a double spatiotemporal localization on a finite continuous background. Starting from this continuous background, it develops progressively towards reaching a climax worth 3 times the level of the background field and then vanishes completely as if it had never occurred [12]. This feature explains well the formation of rogue-wave events [13-18], which have a high amplitude and may appear from nowhere and disappear without a trace [19].

Recent studies reveal that the PS is a universal fundamental solution, characteristic of scalar nonlinear integrable systems such as the celebrated NLS equation [20] and also of vector ones such as Manakov systems [21]. In the latter case, due to the energy transfer between different components, the central amplitude of the vectorial PS can be varied from zero to triple that of the background [22-25]. This is markedly different from the PS in scalar systems, which always has a fixed peak amplitude $[20,26]$. As a special case, the rogue wave that falls to zero in the dip center is usually referred to as a black (or, loosely, a dark) rogue wave [22,24,27], the counterpart of the usual bright PS that has also been observed lately [28]. More interestingly, a frequency-chirped version of the PS, or chirped PS [26], was unequivocally shown to exist in nonlinear media exhibiting a self-steepening effect, which features a doubly localized chirp besides the inherited threefold-amplitude hallmark.

However, there is more and more numerical and experimental evidence that a single rogue-wave event can appear with a peak amplitude higher than 3 (when normalized to the background) [20,29-32]. Such ultrahigh rogue-wave amplitudes are generally attributed to the collisions of several Peregrine breathers [30-32], yielding field profiles that can match well higher-order rational solutions [20]. Now, a fundamental question occurs: Is it possible that a single anomalous PS exists with a peak amplitude more than triple the background height? If so, would such an anomaly possess enough robustness to be observed in a realistic environment, amid the onset of modulation instability (MI)?

In this Letter, we present a first-ever systematic study addressing this question. We clearly show that a PS solution, still expressed by a rational function of second degree, can surprisingly reach an amplitude limit as high as 5 times the background level, in a multicomponent system. Moreover, the interaction of such two PS states can create a spikelike rogue wave of extremely high peak amplitude (nearly 15-fold for certain given parameters). Our result is different from the deterministic colliding events of ordinary solitons [33-35], which could also yield a hump of very high amplitude, yet predictable. We confirm numerically that these high-amplitude PS solutions are robust and can be excited amid the onset of MI. This finding may help shed more light on the anomalous dynamics of rogue waves in other complex multicomponent systems [36].

For our study, we consider an integrable extension of the Manakov system [37], expressed in a normalized form as 
$i D_{\tau} \frac{\partial u_{1}}{\partial \xi}+\frac{\sigma}{2} \frac{\partial^{2} u_{1}}{\partial \tau^{2}}+\left(2\left|u_{1}\right|^{2}+\left|u_{2}\right|^{2}\right) D_{\tau} u_{1}+u_{1} u_{2}^{*} D_{\tau} u_{2}=0$,

$i D_{\tau} \frac{\partial u_{2}}{\partial \xi}+\frac{\sigma}{2} \frac{\partial^{2} u_{2}}{\partial \tau^{2}}+\left(2\left|u_{2}\right|^{2}+\left|u_{1}\right|^{2}\right) D_{\tau} u_{2}+u_{2} u_{1}^{*} D_{\tau} u_{1}=0$,

where $u_{1,2}(\xi, \tau)$ are the complex envelopes of the two field components, with $\xi$ and $\tau$ the distance and retarded time, respectively. $D_{\tau}=1+i \epsilon(\partial / \partial \tau)$ is the differential operator with respect to $\tau$, and $\epsilon(>0)$ scales the perturbation to the Manakov system, which corresponds to the $\epsilon=0$ case here. The parameter $\sigma(= \pm 1)$ denotes the type of dispersion, i.e., +1 for anomalous dispersion and -1 for normal dispersion. As usual, the asterisk denotes the complex conjugate. This coupled form of nonlinear wave equations is a natural extension of the scalar version [38] obtained separately by Fokas on the mathematical side (using a bi-Hamiltonian method) [39] and by Lenells from the standpoint of physics (starting from Maxwell's equations) [40]; hence it is referred to as the coupled FokasLenells (CFL) equations. As an important generalization, the CFL system includes, besides the group-velocity dispersion and self- and cross-phase modulation terms that constitute the Manakov system, the effects of space-time coupling (through the presence of the differential operator in the first term) [41] and self-steepening (through the presence of the differential operator in the last two terms) [42]. These additional terms arise as corrections to the slowly varying envelope approximation [40], when considering the propagation of few-cycle pulses, a situation which can be met in ultrafast optics as well as in hydrodynamics. Thus, the CFL system is relevant to model the propagation of ultrashort optical pulses in birefringent optical fibers $[40,43,44]$ or crossing sea waves in the open ocean $[3,45]$.

By virtue of the standard Darboux transformation procedure [21,46], a special type of fundamental PS solutions of the CFL Eqs. (1) and (2) can be found to be

$$
u_{1}=u_{10}\left(1+\frac{M}{D}\right), \quad u_{2}=u_{20}\left(1+\frac{N}{D}\right),
$$

where

$$
\begin{aligned}
& M=\frac{2 i \epsilon^{2}}{\nu_{2}}\left(\frac{24\left(2 \nu_{2}-\nu_{1}\right) \kappa \sigma \xi}{l_{1}^{2} l_{2}^{2}}+\frac{\left(\kappa^{2}-3 \nu_{2}^{2}\right) \theta}{\delta \nu_{1}}\right)-\frac{\epsilon^{4} \kappa l_{1} l_{2}}{2 \delta^{2} \nu_{1}^{2} \nu_{2}}, \\
& N=\frac{2 i \epsilon^{2}}{\nu_{1}}\left(\frac{24\left(2 \nu_{1}-\nu_{2}\right) \kappa \sigma \xi}{l_{1}^{2} l_{2}^{2}}-\frac{\left(\kappa^{2}-3 \nu_{1}^{2}\right) \theta}{\delta \nu_{2}}\right)-\frac{\epsilon^{4} \kappa l_{1} l_{2}}{2 \delta^{2} \nu_{2}^{2} \nu_{1}},
\end{aligned}
$$

$D=\theta^{2}+\frac{192 \kappa^{2} \delta^{2} \sigma^{2} \xi^{2}}{l_{1}^{4} l_{2}^{4}}+\frac{\epsilon^{4} \kappa^{2} l_{1} l_{2}}{12 \delta^{2} \nu_{1}^{2} \nu_{2}^{2}}-\frac{i \epsilon^{2} \kappa}{\nu_{1} \nu_{2}}\left(\theta-\frac{24 \delta^{2} \sigma \xi}{l_{1}^{2} l_{2}^{2}}\right)$

with $\nu_{j}=\epsilon \omega_{j}-1, \kappa=\nu_{1}+\nu_{2}, \delta=\nu_{1}-\nu_{2}, l_{1}=\sqrt{3} \delta+i \kappa$, $l_{2}=\sqrt{3} \delta-i \kappa=l_{1}^{*}$, and

$$
\theta=2 \epsilon \tau+\sigma\left[1+\frac{4\left(3 \delta^{2}-\kappa^{2}\right)}{\left(3 \delta^{2}+\kappa^{2}\right)^{2}}\right] \xi .
$$

Here $u_{j 0}(j=1,2)$ are the initial plane-wave seeds

$$
u_{j 0}=a_{j} \exp \left(i k_{j} \xi+i \omega_{j} \tau\right),
$$

defined by their amplitudes $\left(a_{j}\right)$, frequencies $\left(\omega_{j}\right)$, and wave numbers $\left(k_{j}\right)$ through the dispersion relations

$$
k_{1}=2 a_{1}^{2}+\frac{a_{2}^{2} \kappa}{\nu_{1}}+\frac{\sigma\left(\nu_{1}+1\right)^{2}}{2 \epsilon^{2} \nu_{1}}
$$

and

$$
k_{2}=2 a_{2}^{2}+\frac{a_{1}^{2} \kappa}{\nu_{2}}+\frac{\sigma\left(\nu_{2}+1\right)^{2}}{2 \epsilon^{2} \nu_{2}},
$$

where

$$
a_{1}=\frac{2 \sqrt{-2 \sigma \delta^{2} \nu_{1}}}{\epsilon\left(3 \delta^{2}+\kappa^{2}\right)}, \quad a_{2}=\frac{2 \sqrt{-2 \sigma \delta^{2} \nu_{2}}}{\epsilon\left(3 \delta^{2}+\kappa^{2}\right)} .
$$

It follows easily from Eq. (11) that the condition of existence of the PS solutions (3) is $\omega_{j}<1 / \epsilon$ for the anomalous dispersion $(\sigma>0)$ case and $\omega_{j}>1 / \epsilon$ for the normal dispersion $(\sigma<0)$ case.

We find from Eqs. (3)-(6) that the above PS solutions, which consist of quadratic polynomials, each have a central amplitude given below:

$$
\left|u_{1}^{c}\right|=a_{1}\left|1-\frac{6 \nu_{2}}{\kappa}\right|, \quad\left|u_{2}^{c}\right|=a_{2}\left|1-\frac{6 \nu_{1}}{\kappa}\right| .
$$

These central amplitudes, calculated at the origin, can be varied with the parameter $\epsilon$ and with the frequencies $\omega_{1}$ and $\omega_{2}$. For example, as $\omega_{1}=1 / \epsilon-\sigma /\left(3 \epsilon^{3}\right)$ and $\omega_{2}=2 \omega_{1}-1 / \epsilon$, one can obtain a PS peak amplitude for the $u_{1}$ field that triplicates the background height [see Figs. 1(a) and 1(b)]. As $\omega_{1}=1 / \epsilon-\sigma /\left(21 \epsilon^{3}\right)$ and $\omega_{2}=5 \omega_{1}-4 / \epsilon$, the PS peak amplitude of the $u_{1}$ field becomes now 4 times the background height [see Figs. 1(c) and 1(d)]. If we take $\omega_{1}=1 / \epsilon-\sigma /\left(4033 \epsilon^{3}\right)$ and $\omega_{2}=64 \omega_{1}-63 / \epsilon$, the peak amplitude can reach nearly 5 times the background level, as seen in Figs. 1(e) and 1(f). Correspondingly, in the above three cases, the $u_{2}$ field 

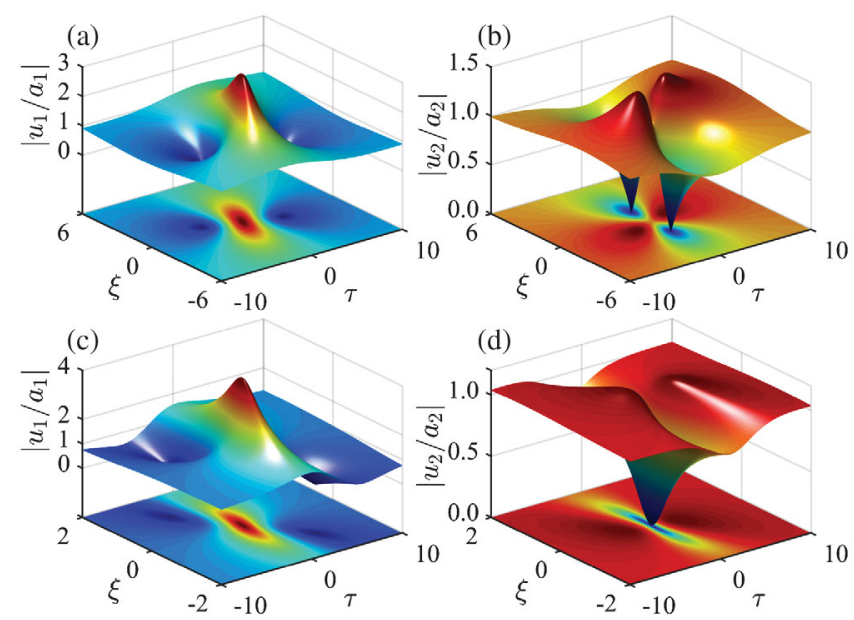

(d)
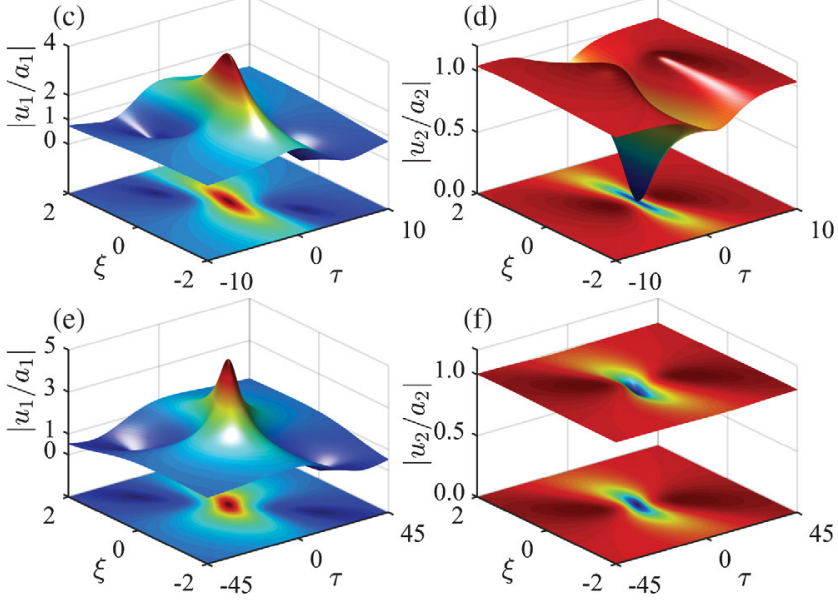

FIG. 1. Surface (top) and contour (bottom) plots of the PS solutions in the anomalous dispersion $(\sigma=1)$ regime, normalized to their respective background heights: (a),(b) $\epsilon=1, \omega_{1}=2 / 3$, $\omega_{2}=1 / 3$; (c), (d) $\epsilon=3 / 5, \omega_{1}=820 / 567, \omega_{2}=320 / 567$; (e), (f) $\epsilon=1 / 5, \omega_{1}=20040 / 4033, \omega_{2}=12165 / 4033$.

would feature an intermediate, a dark, and a near-planar rogue wave, respectively. In either case, the amplitude ratio of the two background fields is given by $a_{2} / a_{1}=\sqrt{\nu_{2} / \nu_{1}}$. Under the amplitude conditions (11), one can verify that the maximum peak-to-background ratio is able to reach 5 , by letting one frequency, say, $\omega_{1}$, approach infinitely to $1 / \epsilon$, whereas letting the other one, say, $\omega_{2}$, be away from it. We should point out that the factor of 5 was usually peculiar to the second-order rogue-wave solutions $[19,20,31,47]$ but now is also achievable in the case of a fundamental PS, contrarily to the common conception developed before. All these properties are exemplified in Fig. 1 for the anomalous dispersion scenario, but they unfold as well with normal dispersion.

The fact that this PS may involve a peak amplitude higher than threefold is very intriguing, as no such case occurs in integrable systems known so far [12,21-25,48]. We attribute this unique amplitude property to the coupling between field components and to the coupling between space and time. Thanks to the former coupling, an energy transfer occurs between different components, so that one component can grow in amplitude at the expense of the other. Meanwhile, due to the space-time coupling in the presence of the self-steepening effect, there occurs a further spatiotemporal rearrangement such that the upper amplitude limit of the usual PS solution can be breached. It is self-evident that the specific conditions for which a more than threefold PS amplitude appears are absent in the Manakov limit $(\epsilon=0)$, where, instead, the peak amplitude for both components is just twice the background height [21].

Moreover, the rational solutions describing the interaction of two such PS states are given by

$$
\begin{aligned}
& u_{1}=u_{10}\left[1-\frac{r s^{*}\left(l_{1}^{3}+l_{2}^{3}\right) / 2 \phi \delta}{2 l_{1}\left(\nu_{1}|s|^{2}+\nu_{2}|w|^{2}\right)-i l_{2}^{2}|r|^{2}}\right], \\
& u_{2}=u_{20}\left[1+\frac{r w^{*}\left(l_{1}^{3}+l_{2}^{3}\right) \phi / 2 \delta}{2 l_{1}\left(\nu_{1}|s|^{2}+\nu_{2}|w|^{2}\right)-i l_{2}^{2}|r|^{2}}\right],
\end{aligned}
$$

where

$$
\begin{aligned}
& r=\gamma_{1}+\gamma_{2} \vartheta-\gamma_{3}\left[\frac{\vartheta^{2}}{2}+\frac{\sqrt{3} \epsilon l_{1}\left(l_{1}-\frac{1}{4} l_{2}^{3}\right) \vartheta-6 \delta l_{1} \tau}{3 \epsilon \delta \nu_{1} \nu_{2}\left(l_{2}^{2}+4\right)}\right], \\
& s=r-\frac{l_{1} \phi}{2 \delta \nu_{1}}\left[\gamma_{2}+\gamma_{3}\left(\frac{\sqrt{3} l_{1}^{2}}{12 \delta \nu_{1} \nu_{2} \phi}-\vartheta\right)\right], \\
& w=r+\frac{l_{1}}{2 \delta \nu_{2} \phi}\left[\gamma_{2}+\gamma_{3}\left(\frac{\sqrt{3} l_{1}^{2} \phi}{12 \delta \nu_{1} \nu_{2}}-\vartheta\right)\right],
\end{aligned}
$$

with

$$
\vartheta=\frac{\tau}{\epsilon}+\frac{\left(l_{2}^{2}+4\right) \sigma \xi}{2 \epsilon^{2} l_{2}^{2}}, \quad \phi=\exp (i \pi / 3)
$$

and $\gamma_{1}, \gamma_{2}$, and $\gamma_{3}(\neq 0)$ being three complex constants. As seen, these solutions, compact yet asymmetrical in form, are composed of polynomials of degree 4 and can reduce to the PS solutions (3) when $\gamma_{3}=0$. They are allowable in either the anomalous or the normal dispersion regime, as seen in Figs. 2(a)-2(d). It is exhibited that the two PS components that constitute a rogue-wave doublet may have a different peak amplitude during interaction; e.g., for the specific parameters used in Fig. 2(a), if one component grows higher than the factor 4 , the other one should be lowered below 4 .

Interestingly, the superposition state of two PS components can reach a very high peak amplitude under a certain parameter condition. A typical example is shown in Figs. 2(e) and 2(f), in which the peak amplitude of the superposed two-PS state can reach nearly 15 times the background, for which the initial plane-wave parameters are the same as in Figs. 1(e) and 1(f), with other parameters being given in the caption. This unusual peak-amplitude feature, corresponding to an intensity factor of around 225 , is actually consistent with the rogue-wave anomaly recently found via a PIC (particle-in-cell) simulations in the gyrotron microwave turbulence, where the rogue waves generated could have an intensity factor of 100-150 [36].

Lastly, one may wonder if these unusual PS solutions could be generated by solving numerically the CFL 

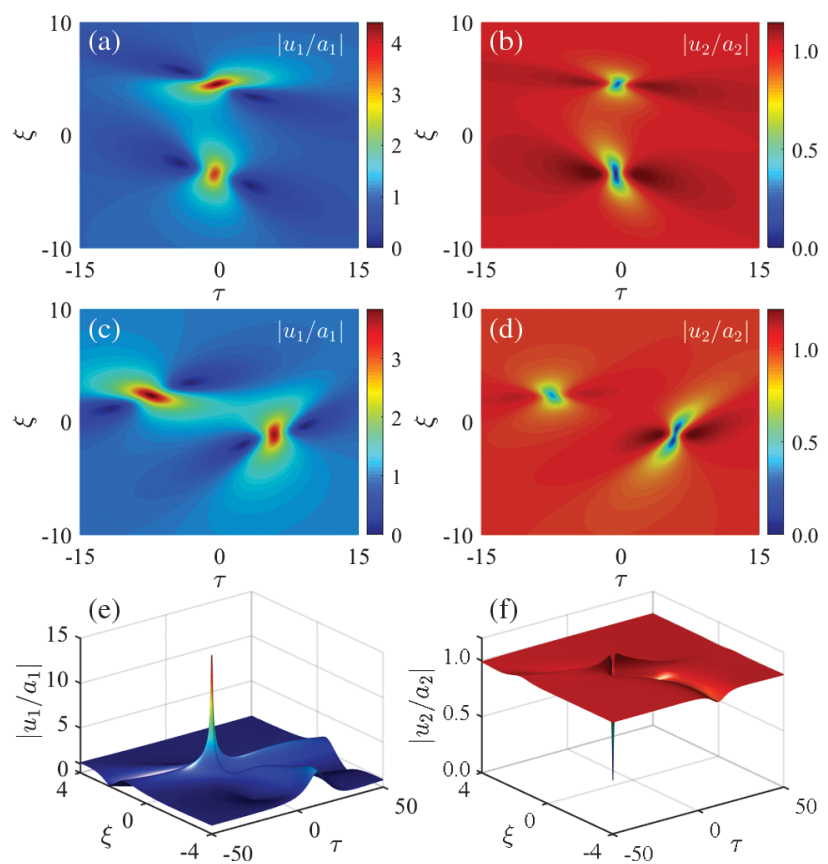

(f)

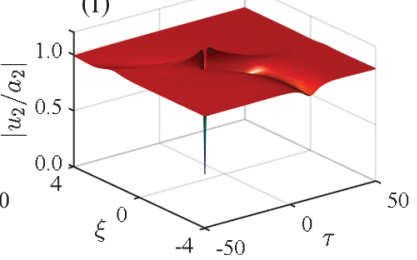

FIG. 2. PS doublets (normalized to background) in either (a),(b) the anomalous dispersion $(\sigma=1)$ or (c),(d) the normal dispersion $(\sigma=-1)$ regime, under the parameter conditions $\epsilon=3 / 5$, $\omega_{1}=1 / \epsilon-\sigma /\left(21 \epsilon^{3}\right), \omega_{2}=5 \omega_{1}-4 / \epsilon, \gamma_{1}=0, \gamma_{2}=-10+10 i$, and $\gamma_{3}=1$. (e), (f) show the formation of extreme rogue-wave spikes with $\gamma_{1}=-1, \gamma_{2}=75 i, \gamma_{3}=1$, and other parameters being the same as in Figs. 1(e) and 1(f).

equations, in the presence of numerical noise that will favor the development of MI. In that prospect, we first inspect the MI gain of the background fields perturbed according to $u_{j}=u_{j 0}\left\{1+p_{j} \exp [-i \Omega(\mu \xi-\tau)]+q_{j}^{*} \exp \left[i \Omega\left(\mu^{*} \xi-\tau\right)\right]\right\}$ $(j=1,2)$, where $p_{j}$ and $q_{j}$ are small parameters and $\Omega$ and $\mu$ are assumed to be positive and complex, respectively, as was done in Ref. [12]. Figure 3(a) shows the logarithmic gain map $\ln \left(\gamma_{h}\right)$, where $\gamma_{h}=\Omega|\operatorname{Im}(\mu)|$, versus $\Omega$ and $\omega_{1}$ for the PS solution of factor 4 , under the specific parameter conditions $\sigma=1, \epsilon=3 / 5$, and $\omega_{2}=5 \omega_{1}-4 / \epsilon$. As $\omega_{1}=820 / 567$, which corresponds to the analytical solution shown in Figs. 1(c) and 1(d), the gain maximum is found to be $\gamma_{h}^{\max } \approx$ 1.026 occurring at $\Omega \approx 0.2$, as indicated by the blue cross in Figs. 3(a) and 3(b). This small gain value suggests that the PS structures of such a kind will be easily generated numerically despite the competing spontaneous MI, and therefore accessible to the experimental observation.

We have numerically solved Eqs. (1) and (2) to reproduce the PS solutions beyond the threefold limit, using the split-step Fourier and spectral methods [49]. Figure 4 shows the simulation results of a fourfold-amplitude PS ( $u_{1}$ field) and a dark PS ( $u_{2}$ field), under the same parameter condition as in Figs. 1(c) and 1(d). It is clear that the whole PS solution is obtained, at least within the region indicated by the white dashed line, perfectly consistent with those shown in Figs. 1(c) and 1(d). Soon
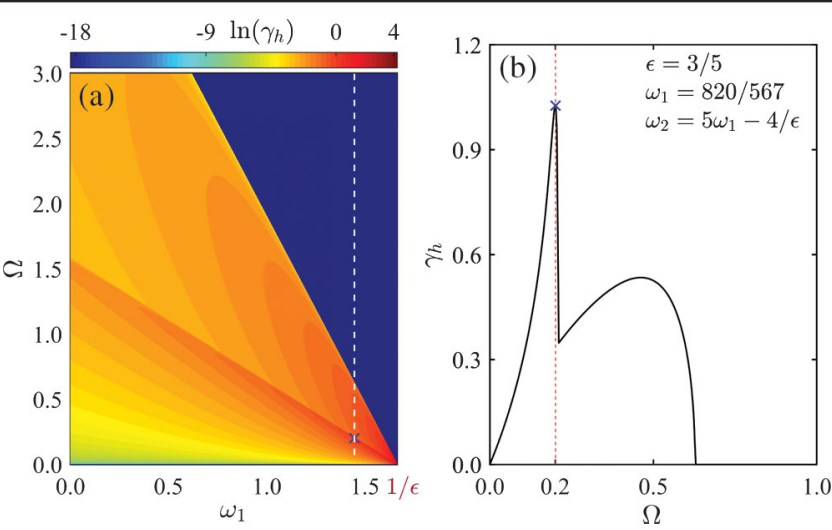

FIG. 3. (a) Logarithmic MI gain map $\left[\ln \left(\gamma_{h}\right)\right]$ of the PS of fourfold amplitude and (b) its gain profile $\gamma_{h}$ at $\omega_{1}=820 / 567$, under the parameter conditions $\sigma=1, \epsilon=3 / 5$, and $\omega_{2}=$ $5 \omega_{1}-4 / \epsilon$. The blue cross corresponds to the case seen in Figs. 1(c) and 1(d), showing a maximum gain $\gamma_{h}^{\max }=1.026$ at $\Omega \approx 0.2$.

afterwards, other periodic waves manifest because of the inherent spontaneous MI; see those occurring at $\xi=4$ in Fig. 4.

Getting closer to realistic experimental conditions, we then confirm numerically that PS solutions featuring an unusual peak-amplitude reinforcement can be excited amid a chaotic background field. To do so, we use the planewave solutions (8) as initial conditions, perturbed by white noise of a strength of $10^{-2}$ [48]. This noisy background subsequently develops into a "sea" of different waves as seen in Figs. 5(a) and 5(b), among which one such typical PS structure could be singled out. For further comparison, the temporal cross-sectional profiles of the numerical solutions selected by white circles at $\xi=18$ are plotted in Figs. 5(c) and 5(d), showing an excellent agreement with the analytical solutions, which confirms our observation above. In addition, one can estimate the period of MI waves occurring at $\xi=5$ to be around $T=80 / 3$, corresponding
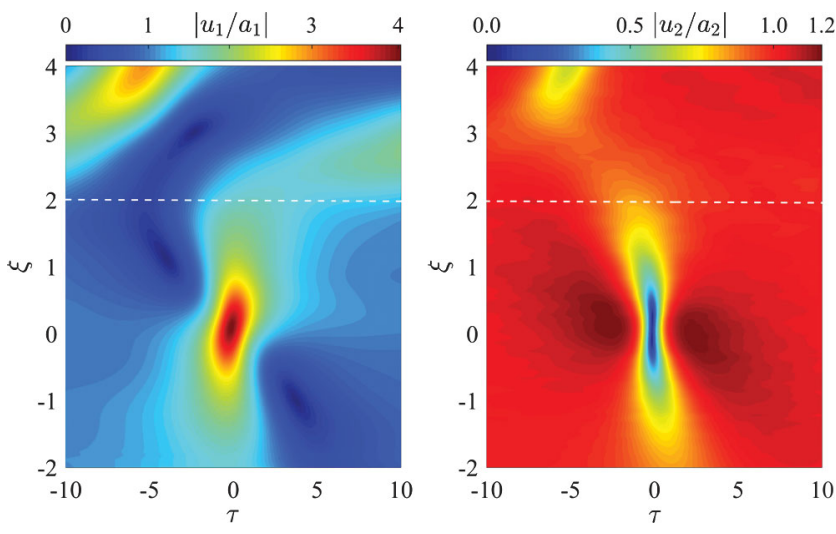

FIG. 4. Simulations of the PS of fourfold amplitude ( $u_{1}$ field) and the dark PS ( $u_{2}$ field) shown in Figs. 1(c) and 1(d), starting from $\xi=-2$ to a longer distance $\xi=4$. 

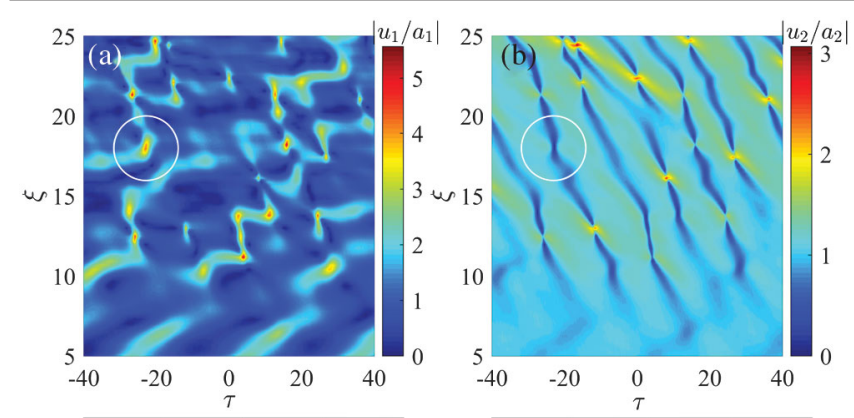

semiconductor planar waveguides [50], and even microwave turbulence [36].

This work was supported by the National Natural Science Foundation of China (Grant No. 11474051) and by the European Union under the European Unions Horizon 2020 research and innovation program MSCA-RISE-2015 (Grant No. 691051). Ph. G. was supported by the Indo-French Centre for the Promotion of Advanced Research (IFCPAR/ CEFIPRA) under Contract No. 5104-2. The work of J. M.
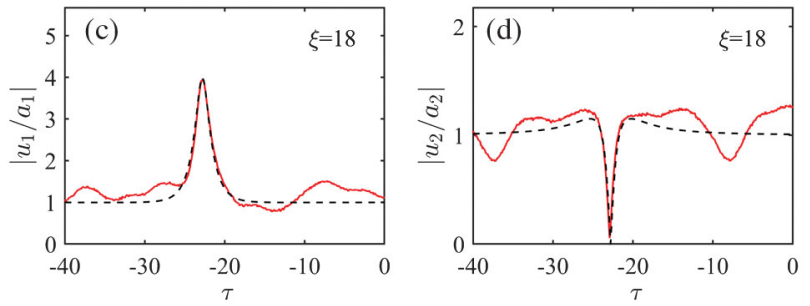

FIG. 5. Numerical excitation of (a) the PS of fourfold amplitude and (b) the dark PS, indicated by the white circles, from a chaotic background field, under otherwise identical parameter conditions as in Figs. 1(c) and 1(d). (c), (d) show their temporal crosssectional profiles at $\xi=18$ (red curves) as compared to our analytical solutions (black dashed curves).

to a modulation frequency of $\Omega=2 \pi / T \simeq 0.23$, which is also well consistent with our MI analysis shown in Fig. 3.

In conclusion, we reported the discovery of a novel rogue-wave anomaly in the context of first-order PS solutions of integrable multicomponent systems. This contrasts sharply with the generally acknowledged maximum of threefold peak amplitude associated to the usual PS solutions, which was ubiquitous among the various integrable models used to date. The salient original features of our work can be exposed as follows. We have obtained explicit analytical rogue-wave solutions of the CFL equations, comprising the PS solutions as well as their twosoliton interaction solutions. Owing to the spatiotemporal coupling of the two involved waves in the presence of the self-steepening effect, the PS solutions can reach a peak amplitude between 3 and 5 times the background level, according to the parameters used. We have supported these analytical predictions by numerical simulations of the propagation model. This was illustrated by the excitation of a fourfold-amplitude PS in the presence of realistic noise on a continuous background. The latter subsequently developed into a chaotic field among which the PS structure could be singled out, as a robust pattern, therefore anticipating the possibility of experimental observation. Such an anomaly of rogue-wave creation is not an exception but a manifestation of novel physical mechanisms that take place in complex multicomponent nonlinear systems, resulting in an extreme wave event of higher amplitude [36]. We expect that our work may spark significant research interest in generation of rogue waves as well as their anomalies in birefringent fibers [44], crossing sea states [3,45], No. TEC2015-71127-C2-1-R and by the Comunidad Autonoma de Madrid (CAM) under Contract No. S2013/ MIT-2790.

*cshua@seu.edu.cn

fabio.baronio@unibs.it

[1] V. I. Shrira and V. V. Geogjaev, J. Eng. Math. 67, 11 (2010).

[2] A. Tikan, C. Billet, G. El, A. Tovbis, M. Bertola, T. Sylvestre, F. Gustave, S. Randoux, G. Genty, P. Suret, and J. M. Dudley, Phys. Rev. Lett. 119, 033901 (2017).

[3] M. Onorato, S. Residori, U. Bortolozzo, A. Montina, and F. T. Arecchi, Phys. Rep. 528, 47 (2013).

[4] J. M. Dudley, F. Dias, M. Erkintalo, and G. Genty, Nat. Photonics 8, 755 (2014).

[5] Nonlinear Guided Wave Optics: A Testbed for Extreme Waves, edited by S. Wabnitz (Institute of Physics, Bristol, 2017).

[6] D. H. Peregrine, J. Aust. Math. Soc. Series B, Appl. Math. 25, 16 (1983).

[7] B. Kibler, J. Fatome, C. Finot, G. Millot, F. Dias, G. Genty, N. Akhmediev, and J. M. Dudley, Nat. Phys. 6, 790 (2010).

[8] A. Chabchoub, N. P. Hoffmann, and N. Akhmediev, Phys. Rev. Lett. 106, 204502 (2011).

[9] H. Bailung, S. K. Sharma, and Y. Nakamura, Phys. Rev. Lett. 107, 255005 (2011).

[10] A. Chabchoub, Phys. Rev. Lett. 117, 144103 (2016).

[11] Yu. S. Kivshar and G. P. Agrawal, Optical Solitons: From Fibers to Photonic Crystals (Academic, San Diego, 2003).

[12] S. Chen, F. Baronio, J. M. Soto-Crespo, Ph. Grelu, and D. Mihalache, J. Phys. A 50, 463001 (2017).

[13] D. R. Solli, C. Ropers, P. Koonath, and B. Jalali, Nature (London) 450, 1054 (2007).

[14] C. Lecaplain, Ph. Grelu, J. M. Soto-Crespo, and N. Akhmediev, Phys. Rev. Lett. 108, 233901 (2012).

[15] P. Suret, R. E. Koussaifi, A. Tikan, C. Evain, S. Randoux, C. Szwaj, and S. Bielawski, Nat. Commun. 7, 13136 (2016).

[16] A. Toffoli, D. Proment, H. Salman, J. Monbaliu, F. Frascoli, M. Dafilis, E. Stramignoni, R. Forza, M. Manfrin, and M. Onorato, Phys. Rev. Lett. 118, 144503 (2017).

[17] A. Safari, R. Fickler, M. J. Padgett, and R. W. Boyd, Phys. Rev. Lett. 119, 203901 (2017).

[18] A. Mussot, C. Naveau, M. Conforti, A. Kudlinski, F. Copie, P. Szriftgiser, and S. Trillo, Nat. Photonics 12, 303 (2018).

[19] N. Akhmediev, A. Ankiewicz, and M. Taki, Phys. Lett. A 373, 675 (2009).

[20] N. Akhmediev, A. Ankiewicz, and J. M. Soto-Crespo, Phys. Rev. E 80, 026601 (2009). 
[21] S. Chen and D. Mihalache, J. Phys. A 48, 215202 (2015).

[22] F. Baronio, A. Degasperis, M. Conforti, and S. Wabnitz, Phys. Rev. Lett. 109, 044102 (2012).

[23] F. Baronio, M. Conforti, A. Degasperis, and S. Lombardo, Phys. Rev. Lett. 111, 114101 (2013).

[24] S. Chen, Ph. Grelu, and J. M. Soto-Crespo, Phys. Rev. E 89, 011201(R) (2014).

[25] S. Chen, F. Baronio, J. M. Soto-Crespo, Ph. Grelu, M. Conforti, and S. Wabnitz, Phys. Rev. A 92, 033847 (2015).

[26] S. Chen, F. Baronio, J. M. Soto-Crespo, Y. Liu, and Ph. Grelu, Phys. Rev. E 93, 062202 (2016).

[27] F. Baronio, M. Conforti, A. Degasperis, S. Lombardo, M. Onorato, and S. Wabnitz, Phys. Rev. Lett. 113, 034101 (2014).

[28] B. Frisquet, B. Kibler, Ph. Morin, F. Baronio, M. Conforti, G. Millot, and S. Wabnitz, Sci. Rep. 6, 20785 (2016).

[29] A. Ankiewicz, J. M. Soto-Crespo, M. A. Chowdhury, and N. Akhmediev, J. Opt. Soc. Am. B 30, 87 (2013).

[30] J. M. Soto-Crespo, N. Devine, and N. Akhmediev, Phys. Rev. Lett. 116, 103901 (2016).

[31] A. Chabchoub, N. Hoffmann, M. Onorato, and N. Akhmediev, Phys. Rev. X 2, 011015 (2012).

[32] M. Närhi, B. Wetzel, C. Billet, S. Toenger, T. Sylvestre, J.-M. Merolla, R. Morandotti, F. Dias, G. Genty, and J. M. Dudley, Nat. Commun. 7, 13675 (2016).

[33] A. V. Slunyaev and E. N. Pelinovsky, Phys. Rev. Lett. 117, 214501 (2016).

[34] Y.-H. Sun, Phys. Rev. E 93, 052222 (2016).

[35] S. Chen, Y. Zhou, F. Baronio, and D. Mihalache, Rom. Rep. Phys. 70, 102 (2018).
[36] N. S. Ginzburg, R. M. Rozental, A. S. Sergeev, A. E. Fedotov, I. V. Zotova, and V. P. Tarakanov, Phys. Rev. Lett. 119, 034801 (2017).

[37] M. X. Zhang, S. L. He, and S. Q. Lv, J. Nonlinear Math. Phys. 22, 144 (2015).

[38] S. Chen and L.-Y. Song, Phys. Lett. A 378, 1228 (2014).

[39] A. S. Fokas, Physica D (Amsterdam) 87, 145 (1995).

[40] J. Lenells, Stud. Appl. Math. 123, 215 (2009).

[41] R. W. Boyd, Nonlinear Optics, 3rd ed. (San Diego, Academic, 2008), Chap. 13.

[42] J. Moses and F. W. Wise, Phys. Rev. Lett. 97, 073903 (2006).

[43] P. K. A. Wai, C. R. Menyuk, and H. H. Chen, Opt. Lett. 16, 1231 (1991).

[44] F. Baronio, B. Frisquet, S. Chen, G. Millot, S. Wabnitz, and B. Kibler, Phys. Rev. A 97, 013852 (2018).

[45] M. Onorato, A. R. Osborne, and M. Serio, Phys. Rev. Lett. 96, 014503 (2006).

[46] Y. Zhang, J. W. Yang, K. W. Chow, and C. F. Wu, Nonlinear Anal.: Real World Appl. 33, 237 (2017).

[47] P. Dubard, P. Gaillard, C. Klein, and V. B. Matveev, Eur. Phys. J. Spec. Top. 185, 247 (2010).

[48] S. Chen, Y. Ye, F. Baronio, Y. Liu, X.-M. Cai, and Ph. Grelu, Opt. Express 25, 29687 (2017).

[49] F. Baronio, S. Chen, Ph. Grelu, S. Wabnitz, and M. Conforti, Phys. Rev. A 91, 033804 (2015).

[50] J. U. Kang, G. I. Stegeman, J. S. Aitchison, and N. Akhmediev, Phys. Rev. Lett. 76, 3699 (1996). 